\title{
Pratiques
}

Linguistique, littérature, didactique

$179-180$ | 2018

Poésie et langue : aspects théoriques et didactiques

\section{Le poème : habiter la métropole parisienne}

The poem: living in the Paris metropolis

\section{Sandrine Bédouret-Larraburu et David Bédouret}

\section{(2) OpenEdition}

Journals

Édition électronique

URL : http://journals.openedition.org/pratiques/5461

DOI : 10.4000/pratiques.5461

ISSN : 2425-2042

Éditeur

Centre de recherche sur les médiations (CREM)

\section{Référence électronique}

Sandrine Bédouret-Larraburu et David Bédouret, « Le poème : habiter la métropole parisienne »,

Pratiques [En ligne], 179-180 | 2018, mis en ligne le 31 décembre 2018, consulté le 19 avril 2019. URL: http://journals.openedition.org/pratiques/5461; DOI : 10.4000/pratiques.5461

Ce document a été généré automatiquement le 19 avril 2019.

(c) Tous droits réservés 


\section{Le poème : habiter la métropole parisienne}

The poem: living in the Paris metropolis

Sandrine Bédouret-Larraburu et David Bédouret

\section{Introduction}

1 Depuis la réforme de 2016, le concept d'habiter est au cœur des programmes de géographie de cycle 3. Il prend acte d'une évolution épistémologique importante qui met au centre des réflexions le sensible, l'idéel, la géographicité. Il favoriserait l'apprentissage d'un raisonnement complexe et la compréhension des différentes dimensions d'un territoire (matérielle, culturelle et phénoménologique). Les enseignants doivent réinventer leur approche et leurs pratiques pour appréhender ce monde invisible du sensible et la relation existentielle établie entre l'homme et son habitat. Le poème est alors un allié de poids, pour capter et pour comprendre les relations qu'entretiennent l'homme et l'espace, car il est une expérience de vie.

2 Rappelons qu'H. Meschonnic (1982) définit le poème comme l'action d'une forme de vie sur le langage et inversement. Il s'agira alors de montrer comment le langage poétique, le paysage et l'expérience sensible interagissent. Nous avons choisi l'objet géographique de la ville, et plus particulièrement Paris. En effet, les programmes de géographie préconisent l'étude d'une métropole et cette capitale culturelle a inspiré de nombreux poètes. Le corpus est composé de dix poèmes publiés entre 1999 et 2018 pour que le rapport à la ville soit perçu comme contemporain ${ }^{1}$. Il permet d'assurer une variété de formes et présente un groupement thématique autour du canal et de la porte SaintMartin dans le $\mathrm{X}^{\mathrm{e}}$ arrondissement : les deux poèmes de J. Roubaud et celui d'O. Barbarant autorisent une confrontation thématique intéressante. De plus, ce corpus peut se lire à partir de trois entrées liées à la perception du monde sensible :

- Le rapport au mouvement et à l'immobilité : comment se place l'énonciateur ? Se place-t-il dans le mouvement de la ville ? Ou en est-il extérieur ? En quoi ce positionnement influe-t-il sur le rythme du poème, comme inscription d'un sujet et d'une signifiance? 
- Le rapport à la ville comme espace urbain, humanisé ou de façon plus surprenante ou inattendue, comme espace de nature.

- Le rapport à la littérature et aux représentations que l'on peut avoir de la ville, de Paris ici, par sa propre culture. propres logiques et leurs propres outils. Elles appartiennent à des «communautés discursives " différentes, ce qui entraine des incompréhensions et des malentendus (Bernié, 2002 ; Jaubert, 2007). Elles utilisent du vocabulaire spécifique parfois même des mots identiques pour désigner des réalités différentes. Dans le cadre scolaire, cela entraine un cloisonnement disciplinaire dans lequel les élèves naviguent alors même que les frontières des disciplines universitaires se redessinent. Ainsi, dès 1989, le rapport Bourdieu-Gros préconise «d'échapper à la simple comptabilité par discipline » afin d'éviter une sclérose des savoirs. En effet, les apprenants doivent acquérir ces différentes formes de langages sans forcément les croiser, ce qui ne favorise pas la compréhension de processus complexes. Dans ce contexte, dès les années 1980, l'Éducation nationale favorise la coopération entre les disciplines scolaires (Vergnolle-Mainar, 2011) et elle promeut l'interdisciplinarité. Les dispositifs se succèdent en collège et en lycée : parcours diversifiés, travaux croisés, itinéraires de découverte, travaux personnels encadrés, enseignements pratiques interdisciplinaires.

L'étude de Paris à partir d'un même corpus de poèmes contemporains effectuée par un géographe et une poéticienne témoigne de ces deux communautés discursives, alors que leurs approches peuvent être complémentaires. Il ne s'agit pas de créer une nouvelle discipline, une « géopoétique » (Collot, 2014) mais de créer un partage de signification et de faire résonner géographie et poétique pour enrichir les deux domaines. La posture du poète et celle du géographe diffèrent mais elles s'allient pour faire surgir le complexe. Cette double réflexion permet en particulier au niveau didactique et pédagogique de construire des savoirs hybrides s'appuyant à la fois sur des compétences géographiques et littéraires.

Ainsi nous voudrions montrer qu'au sein d'un dispositif interdisciplinaire, appréhender la signifiance du poème, au sens d'H. Meschonnic, c'est-à-dire de l'inscription d'un sujet dans un rythme, permettrait d'éclairer de manière plus complexe la notion d'habiter, en tant que concept-clé de géographie.

6 Cette réflexion est structurée en trois parties : une approche géographique faisant le lien entre poésie et habiter; une approche poétique et stylistique des poèmes sur la ville et enfin une proposition didactique et pédagogique, centrée sur l'interdisciplinarité.

\section{Les poèmes témoignent de la géographicité du poète et d'une forme d'habiter la métropole parisienne}

\section{Habiter et poésie ${ }^{2}$}

7 La force du concept d'habiter est de concilier les dimensions matérielles et idéelles du territoire et il prend en compte toutes les grandes mutations épistémologiques qu'a connues la géographie ces dernières années. Si les géographes ont de nombreux outils à leur disposition pour approcher l'aspect physique de l'espace: carte, image satellite, Système d'information géographique (SIG)3 , données statistiques, etc., ils ont développé 
aussi des démarches qualitatives pour mesurer les données sociales et culturelles comme l'enquête ou encore l'entretien conversationnel semi-directif. Des ateliers-promenades sont même mis en place pour mesurer le rapport sensible qu'entretiennent les hommes avec les lieux (Manola, Bailly \& Duret, 2017). L'imaginaire est aussi un champ d'investigation en expansion, car il est perçu comme une dynamique par laquelle un sujet entre en contact avec le monde, y œuvre et s'y construit (Lhérété, 2015). L'utilisation de l'imaginaire permet donc d'accéder à " des réalités masquées des territoires et des lieux ", il est un " détour pour appréhender le réel », car il est un "processus de recomposition, de recréation du monde par l'intermédiaire d'images, de symboles, de signes, de formes, de représentations qui assurent aux sociétés, à l'individu, au sujet une médiation fondamentale avec les lieux, l'espace dans sa complexité »(Dupuy \& Puyo, 2015).

8 Le cinéma, la littérature, les œuvres fictionnelles sont alors utilisés par les géographes comme des instruments d'exploration du monde car ils traduisent l'expérience d'une dimension existentielle de l'espace géographique. «Or, la connaissance et la compréhension de cette expérience de l'espace sont bien une dimension majeure de la géographie que l'on peut appeler "géographie de l'expérience" "(Desbois, GervaisLambony \& Musset, 2016). Ainsi, la poésie nous plonge dans l'imaginaire du poète, dans son expérience des lieux, dans son être au monde. Cet imaginaire n'est pas synonyme d'irréel mais il est l'ancrage phénoménologique qui lie chaque homme au monde matériel. Le langage devient le moyen de comprendre l'invisible : les ambiances qu'elles soient sonores, olfactives, tactiles ou encore les perceptions du temps et de l'espace. En cela la poésie témoigne de la présence au monde du poète, de sa géographicité c'est-à-dire de son être au monde. De fait la poésie fait surgir l'habiter soit le processus de construction des individus et des sociétés par l'espace et de l'espace par l'individu, en d'autres termes elle témoigne de l'expérience physique et mentale d'un lieu, voire un rapport ontologique qu'entretient chaque individu à son espace.

\section{Des poètes géographes face aux paysages urbains}

9 Les poètes de notre corpus centrent leur texte sur les paysages, vus comme un élément de la découverte de la réalité géographique. Ceux-ci sont conçus comme une portion de l'espace que l'on embrasse du regard. Il en découle une mise à distance où le poète se place en observateur et applique une démarche géographique traditionnelle : il localise, situe, décrit afin de dégager les éléments structurants du paysage. Les titres des poèmes indiquent souvent un lieu: canal Saint-Martin, Oberkampf pour J. Roubaud; place Fontenoy et rue Sorbier pour J. Réda ; jardin des plantes pour R. Marteau, ou encore la porte Saint-Martin pour O. Barbarant. Ce besoin de localiser et de situer est encore plus marqué chez J. Roubaud dans "Canal Saint-Martin» (Roubaud, 1999, p. 108) qui commence son poème par donner l'emplacement de l'action, sa propre localisation et ce qu'il regarde. Il fixe des points et trace des lignes avec son regard, comme s'il plaçait des coordonnées géographiques avec une longitude et une latitude : « d'un côté du canal c'est le quai de Valmy ; Et de l'autre côté c'est le quai de Jemmapes ; [...] sur un banc [...] en face de l'écluse » (ibid.). Dans « Sunday, mein Oberkampf » (ibid., p. 109), il arpente le territoire comme le géographe, il énumère les noms des rues et des magasins, il va de point en point et délimite ainsi le territoire de sa promenade urbaine (figure 1). 

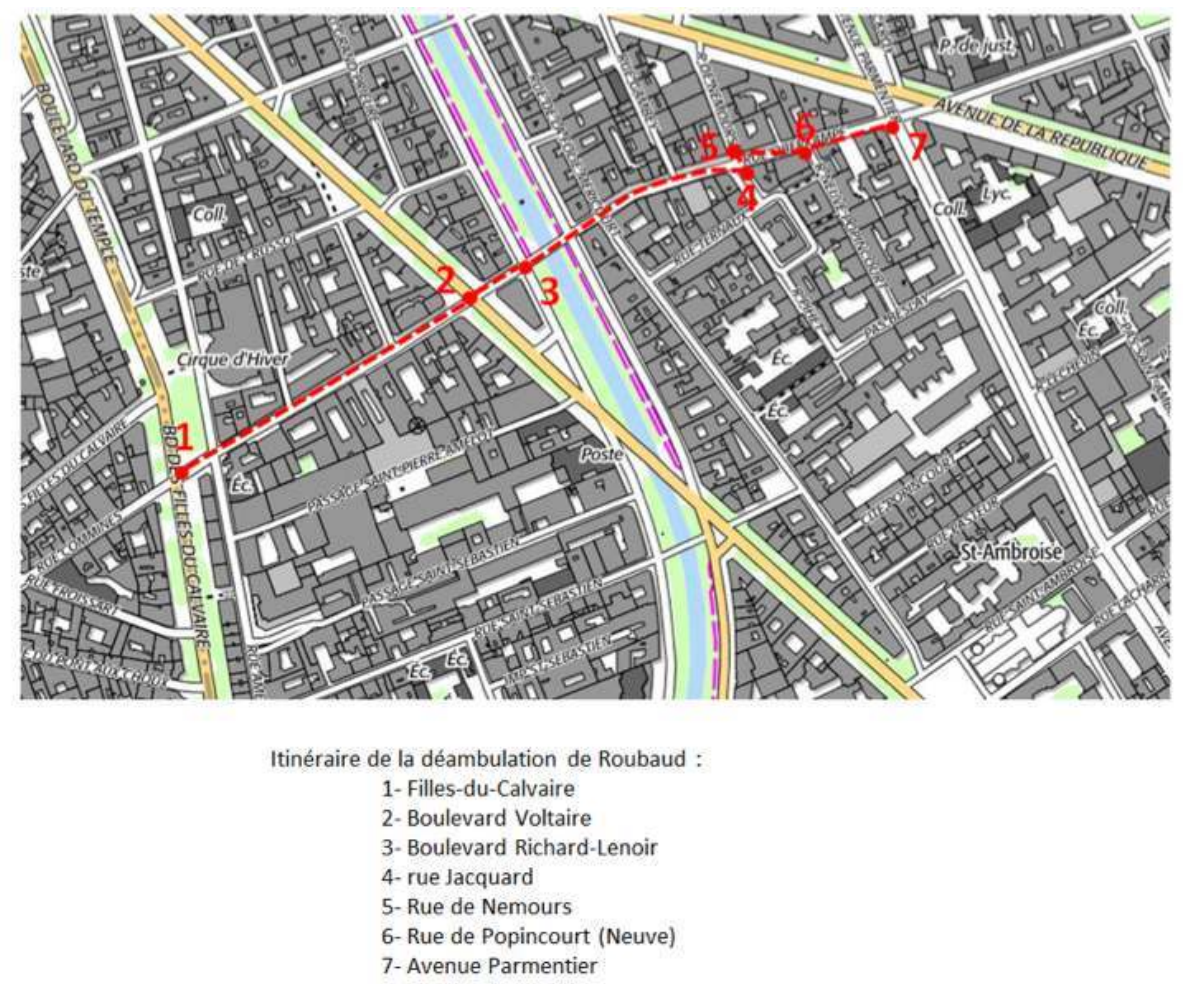

À la description spatiale s'ajoute souvent une temporalité ; la description est datée : « Un soir désert en avril à Paris » (« Marronniers, place Fontenoy », Réda, 2004, p. 100); « Vers sept heures, le square [...]» («Gamines, square Sorbier», Réda, 2004, p. 103-104). R. Marteau ne met pas de titres mais il date et localise chaque poème, ce qui crée un effet littéraire générique (carnet de voyage) : «Jardin des Plantes, samedi $1^{\text {er }}$ décembre 2001 » (Marteau, 2012, p. 165). J. Roubaud indique même les saisons pour chaque strophe dans «Canal Saint-Martin » (Octogone, 2014, p 27-28), il fait se succéder l'été, l'automne, l'hiver et le printemps, ce qui lui permet d'observer les transformations du paysage, à la manière du géographe qui utilise la technique des photographies répétées. Les poètes mettent en place un chronotope dans lequel la ville se structure autour de lieux et de temporalités. Toutefois, le langage poétique peut témoigner d'une autre posture géographique dans laquelle le poète se place non plus hors du paysage mais dans le paysage qui devient une expérience sensorielle. Les ambiances sont retranscrites surtout par la vue mais aussi par l'ouïe. J. Roubaud capte les bruits de l'eau, des moteurs ; J. Réda est plus sensible aux "chuchotements du vent» rue Sorbier alors que R. Marteau semble subjugué par les sifflements d'oiseaux au jardin des plantes. Les poèmes témoignent du rapport sensible qu'il y a entre l'habitant et son lieu de vie.

12 Le fait de se positionner dans le paysage déclenche une multitude d'émotions et le paysage devient un état d'âme. J. Réda (2004, p. 100), place Fontenoy, est littéralement connecté à la nature ; ce qui provoque un débordement émotionnel :

Cinq arbres me tenaient très doucement

Comme une main d'ombre sans tremblement

D'amour, de joie ou de colère 

avoir «bu une semaine de boulot et de sale temps ». O. Barbarant (2015, p. 151-152) lui aussi nous dépeint le stéréotype de la capitale bétonnée, grise, bruyante, rythmée par la circulation : " le serpentement monstrueux de la circulation; les feux des phares qui s'y écrasent à rythme régulier; tumulte des moteurs relancés ou force crissements des freins ; deux arches sales ». Il insiste même sur l'aspect populeux et cosmopolite avec ses " géants africains » et avec ses magasins exotiques dont les noms évoquent leur origine : «Cantal, Italie, Bosphore », ce qui fait écrire au poète que « la géographie [est] devenue insensée » (ibid., p. 152).

18 J. Roubaud nous rappelle l'importance de la fonction marchande des rues parisiennes, en énumérant tous les magasins rencontrés sur son parcours : crêperie, lingerie, vaisselles, jouets, pressing, parfum, bar, café. Il montre que cet espace est en mouvement, que les enseignes évoluent au gré de la conjoncture économique. En effet, quinze ans plus tard, il récrit le poème intitulé cette fois-ci « Sunday, mine Oberkampf » (Octogone), il fait un état des lieux des magasins : « la crêperie Morgane (disparue) ; lingerie Fabiola (toujours là)» (Roubaud, 2014, p. 27).

19 Paris est une ville touristique avec des lieux remarquables : l'école militaire, le canal Saint-Martin, Belleville, le jardin des plantes, Montmartre, la Butte-aux-cailles, la Seine. Elle est aussi un espace vécu, un territoire du quotidien, un lieu approprié par les populations. Les poètes prennent ainsi des instants de vie. J. Réda (2004, p. 103) observe un groupe d'adolescentes qui fument à Belleville : « Elles viennent fumer à quatre sur un banc »; " elles fument toujours, parlent en s'esclaffant». Tandis que S. Ritman (2017, p. 292) est témoin de «l'eau de l'Oise [qui] monte sur les berges du RER deux ivrognes descendent à Neuville-Université »; ou encore quelques passants traversent les poèmes : «tu traverseras la passerelle » (Roubaud, 1999, p. 108) ; "piétons ailés » (Marteau, 2012, p. 165) ; « parallélogrammes de piétons » (Roubaud, 2014, p. 28 ) ; «invitent les passantes à se rendre dans l'un des nombreux salons de coiffure » (Barbarant, 2015, p. 152). 
Toutefois, la poésie nous offre un autre regard sur Paris et elle permet de déconstruire les représentations que nous pouvons avoir de la capitale en particulier en effaçant l'opposition ville nature. Les poètes montrent que la nature est présente en ville. Le paysage urbain de J. Réda (2014, p. 100) «près de l'école militaire » se compose de « cinq marronniers fleuris » et s'organise « entre les fleurs, les feuilles et la terre ». R. Marteau va plus loin car il efface les éléments humains pour concentrer son regard sur le végétal et l'animal. Le poète est spectateur de cette nature où l'homme est remplacé par les oiseaux : corneilles, palombes, étourneaux, pigeons sont les acteurs de ses vers, «Piétons ailés oublieux de l'air et du ciel » (Marteau, 2012, p. 165). Alors qu'il regarde Paris « des hauteurs de Montmartre ou de la Butte-aux-cailles» (ibid., p. 188) il ne retient que le coucou, le bois, les feuilles, les fleurs, les branches, le cerisier, le vent, la Seine. Pourtant de ces lieux, il devrait voir les toits, les axes de communication, les monuments, les hommes. Il dénonce ce monde urbain où la société est devenue « un peuple perdu » (ibid.), tout en faisant une apologie de la nature.

Enfin, Paris est inscrite dans l'imaginaire individuel et collectif, elle reste la ville lumière avec ces lieux symboliques. Le poème de V. Rouzeau rend hommage à cet imaginaire en faisant un collage d'extraits d'Alcools de G. Apollinaire qui a fait du pont Mirabeau le symbole des amours perdues. "Cous coupés » est le dernier vers de "Zone », «Serais-tu la Ville de Paris » fait écho aux voix des villes de «Vendémiaire »; «Et où coule ta peine qu'il toujours t'en souvienne » est un collage de quelques vers du « Pont Mirabeau ». C'est dire là la force du poème quand il parvient à modifier la perception de la réalité de la ville. Les vers de G. Apollinaire deviennent plus signifiants que l'objet lui-même.

Par conséquent, ce corpus nous montre que Paris est une expérience polytopique et sensible car les individus sont géographiquement pluriels (Stock, 2004). En d'autres termes, Habiter Paris ne se résume pas à se loger, circuler, travailler dans une ville mondiale mais c'est faire l'expérience de la vie dans différents lieux. Ce polytopisme indique aussi des significations multiples d'un même lieu car l'investissement et les pratiques sont différents selon les individus et les groupes sociaux. La poésie permet alors cette prise de conscience de son inscription dans le temps et dans l'espace et elle met en exergue les manières d'habiter les lieux géographiques.

\section{Une expérience de vie en interaction avec une expérience de langage}

Le poème offre une mise en mots singulière qui doit permettre au lecteur d'appréhender différemment l'espace dans lequel il vit.

\section{L'inscription du réel}

24 Les poèmes du corpus s'inscrivent dans un espace choisi et mentionné. Si le poète choisit d'inscrire le hic de l'événement, il cherche aussi à ouvrir une brèche dans ce quotidien décrit. Il cherche par le langage à construire un autre point de vue. Ainsi, J. Roubaud (1999, p. 108), après avoir présenté le paysage, introduit une veine lyrique «pendant que du temps fuit ». L'espace de quelques vers, le regard semble se détourner du réel et le langage cherche à saisir une impression fugace : 
L'image dans les eaux se referme en ovale / Pâles platanes flous de feuilles tombées

pâles // S'enfonçant dans le ciel pâle et blanche jusqu'au fond. (ibid.) donc le choix de montrer un Paris autre, moins urbain. Ainsi dans "Marroniers, place Fontenoy ", située dans le septième arrondissement, le poète J. Réda évoque sa rencontre étrange avec cinq marronniers. Le poème prend figure de comptine du fait de l'hétérométrie des vers (un octosyllabe au milieu de décasyllabes dans chaque strophe) et des rimes croisées, la rime féminine en [Er] parcourant l'ensemble du poème. Par hypallage, le paysage prend vie. En vain, le poète cherche une explication à une scène étrange mais repart, léger. L'expérience est ici plutôt gaie, un moment de solitude et de communion, dont le poète s'amuse par le registre et par la structure métrique.

\section{Le mouvement de la ville saisi dans la langue}

La langue poétique donne une vie, une autonomie à la ville, dans laquelle le sujet poétique se dissout. Le sonnet XI de J. Roubaud «Sunday, mein Oberkampf » est exemplaire de ce fonctionnement: le poète décrit précisément un parcours mais c'est le paysage qui 
semble bouger, non pas lui «assis sur un banc boulevard Richard-Lenoir» (Roubaud, 1999, p. 109). Tous les verbes d'action ont un sujet inanimé : « la crêperie jouxte », « la rue Oberkampf tend», «ma route croise » avec le double sens que peut avoir ce syntagme: ma route croise peut indiquer le mouvement du poète, comme il peut indiquer l'objet concret, le bitume, alors que le poète reste assis. Le seul verbe de parole « contredira " a pour sujet «le café ». Le langage poétique rend ici compte de l'activité de la ville indépendamment du regard des gens qui s'y trouvent, perçue dans la subjectivité du poète, qui se laisse submerger par elle.

L'hostilité vis-à-vis du paysage urbain se lit dans l'ode d'O. Barbarant. "Le chant de la porte Saint-Martin» (2015, p 151) comporte deux temps: le premier est consacré à l'intérieur d'un appartement "où ne s'asseyent que des souvenirs »; le sujet poétique $\mathrm{y}$ exprime la douleur de la perte. Le second temps est consacré à l'extérieur; la douleur s'exprime par l'agression que constitue le monde extérieur qui continue d'exister :

J'ai beau écrire que le jour n'a plus le droit de renaître ni de lancer

Sa main pâle et livide à l'arête des toits

Que les vitrines des cafés n'ont plus lieu d'être quand vos reflets n'y passent plus

Les deux portes de pierre noire et jaune enjambant les boulevards

Ne cessent pourtant pas d'y jouer les écluses

De minute en minute arrêtant ou laissant s'écouler

Tantôt le serpentement monstrueux de la circulation

Tantôt les feux des phares qui s'y écrasent à rythme régulier

Chaque vague y jetant l'ordinaire tumulte de moteurs relancés ou force crissement

de freins

À mi-chemin des deux arches sales le bureau de tabac vit sa vie

Des foules se forment qui tard s'émiettent

Devant la bouche du métro des géants africains invitent les passantes à se rendre

Dans l'un des nombreux salons de coiffure poussés un peu partout (ibid.)

31 Ce passage est remarquable par la sensation de mouvement que rend le phrasé. L'isotopie est d'abord très présente: "passent», « enjambant», «jouer», "s'écouler », "serpentement", "circulation", "s'écrasent à rythme régulier», "vague», "se forment ", "s'émiettent ». Le temps se mesure par le mouvement des écluses et le jeu des feux de circulation. L'univers quotidien apparait pourtant hostile du fait des négations : «n'a plus le droit», «n'ont plus lieu», «n'y passent plus», «ne cessent pas » et du lexique dysphorique: «monstrueux, écrasent, jeter, tumulte, s'émiettent»; la porte Saint-Martin devient une sorte de monstre qui engloutit les passants. Ainsi le poète joue également d'une syllepse sur le mot rendre : " les passantes à se rendre ", où rendre peut signifier "abandonner la lutte», ou «se diriger ». L'attente à la fin du vers permet de jouer sur l'ambigüité alors que les salons de coiffure poussent à la place de la végétation.

Pour le poète ici, il s'agit de faire passer l'émotion ${ }^{4}$ par la réalité des faits quotidiens, par l'hostilité de l'extérieur. "À croire que seule demeure / De notre déchirure une géographie devenue insensée ", écrit-il quelques vers plus loin. La géographie qu'évoque O. Barbarant est une écriture des lieux, une objectivisation. Elle devrait permettre d'organiser le monde. En revanche, Barbarant-poète donne à lire une géographie du lieu qui permet de diffracter, d'extérioriser la douleur. Pour ce faire, le poète utilise des vers libres longs. La première strophe citée ci-dessus, qui est en fait la troisième du poème, est composée d'une seule phrase comprenant deux propositions en parataxe, une subordonnée temporelle en hypotaxe à la première proposition, une relative et de nombreux participes présents complétant la seconde. Cette longue phrase cherche son souffle comme si le poète cherchait à respirer, dehors, dans «l'ordinaire tumulte du 
monde ", qui fait figure d'oxymore, caractérisé par le bruit de la circulation, le crissement des pneus et l'agitation des hommes.

Dire le paysage permet alors de plonger à la source des images et de les extérioriser : il ne s'agit pas ici de mettre en mots un instant "volé ", étranger à la ville, mais au contraire de composer avec ce qui en fait son essence.

Le poème est donc interaction d'une forme de vie et d'une forme de langage : le poète peut alors chercher dans la ville son étrangeté ou composer avec ses éléments concrets pour rendre compte d'une expérience; le langage poétique doit néanmoins sortir du signifié : ce sont les rythmes, les figures, les images qui rendent compte d'une expérience unique qui doit agir sur la perception du lecteur, sur sa perception de la ville, mais aussi de la langue. Le poème dit, par son utilisation de la langue, quelque chose de plus sur le paysage: le poète construit un paysage, défini comme espace perçu, dans et par sa subjectivité, nourrie d'un imaginaire collectif. Le poète propose des paysages singuliers dont une approche poétique poussée peut aider le géographe à rendre compte. L'approche poétique doit sortir de la simple signification pour ouvrir sur une signifiance des textes, là où le rythme des poèmes dit autre chose que les signifiés de l'espace posé.

\section{Paris, un objet géographique et poétique pour la classe}

Depuis 2015, les enseignements pratiques interdisciplinaires (EPI) se sont déployés en cycle 4 puis en $6^{\mathrm{e}}$ à partir de la rentrée 2017. Ils représentent une modalité nouvelle de mise en œuvre des programmes disciplinaires. Ils doivent permettre aux enseignants de réaliser une partie de leur programme dans un autre contexte pédagogique et didactique car ils reposent sur l'interdisciplinarité, la pédagogie de projet et le travail de groupe (Bulletin officiel $\mathrm{n}^{\circ} 28$ du 28 mai 2015). L'objectif est de construire et d'approfondir des connaissances et des compétences inscrites dans les programmes d'enseignement à partir d'une réalisation concrète, individuelle ou collective. En classe de $6^{e}$, la séquence sur " habiter une métropole » permettrait de croiser la géographie et le français. En français, l'objet d'étude "Récits de création; création poétique » permet d'investir le champ poétique. Les programmes de 2017 préconisent «des poèmes de siècles différents, célébrant le monde et/ou témoignant du pouvoir créateur de la parole poétique ». Si la perspective se veut synchronique ici, il s'agit bien de montrer comment le pouvoir créateur de la parole poétique enchante ou désenchante la ville de Paris.

Par ailleurs, « le langage oral trouve à se développer dans les dialogues didactiques, dans l'explicitation des démarches, dans les débats de savoirs ou d'interprétation (à propos de textes, d'images ou d'expériences), dans les comptes rendus, dans les présentations orales, dans les discussions à visée philosophique, en lien avec l'enseignement moral et civique » (B.O. spécial $n^{\circ} 11$ du 26 novembre 2015). Il est ainsi intéressant de montrer quelle est la démarche du géographe qui décrit et analyse son environnement, et de la mettre en parallèle avec l'expérience du poète qui cherche à subjectiver par la langue son expérience d'habiter. Cet EPI permet à la fois de réfléchir à la construction de l'espace mais aussi aux différents discours subjectifs ou objectifs qui en résultent. Il doit permettre « la découverte, l'analyse et la compréhension des relations dynamiques que les individus-habitants et les sociétés entretiennent à différentes échelles avec les territoires et les lieux qu'ils pratiquent, conçoivent, organisent, représentent » (B.O. 
spécial $\mathrm{n}^{\circ} 11$ du 26 novembre 2015). Dans le cadre du thème 1 du programme de géographie: «habiter une métropole» et du sous-thème: «les métropoles et leurs habitants ", est préconisée la mise en place d'une étude de cas d'une métropole d'un pays développé. C'est pourquoi, nous proposons d'intituler cette dernière « Habiter Paris, une ville mondiale ». Elle repose uniquement sur un corpus de poèmes et sur l'utilisation de globes virtuels comme Google map et Street view. Nous avons retenu quatre poèmes qui sont à la fois un objet et un outil d'apprentissage : de J. Roubaud, "Canal Saint-Martin " (1999, p. 108) «Sunday, mein Oberkampf » (ibid., p. 109) et «Canal Saint-Martin (2014, p. 27-28) ; enfin d'O. Barbarant "Chant de la porte Saint-Martin» (2015, p. 151-152). Le déroulement pédagogique est synthétisé dans le tableau ci-dessous (tableau 1), il est basé sur un croisement de compétences disciplinaires. Les deux communautés discursives sont ici invoquées dans une même séquence, les activités invitent les élèves à passer de l'une à l'autre, les savoirs se construisent dans ce balancement géographie-poétique ${ }^{5}$. Le travail final est la création d'un carnet rassemblant toutes les productions : poèmes, carte de Paris, carte postale paysagère, schéma de Paris, parcours de J. Roubaud, parcours construit sur globe virtuel, création poétique. Ce carnet est la matérialisation de leur expérience de l'habiter la ville à travers la poésie et de leur expérience de la poésie à travers la ville.

Tableau 1. Séquence pédagogique d'E.P.I. en classe de $6^{\mathrm{e}}$

\begin{tabular}{|c|c|c|}
\hline \multicolumn{3}{|c|}{ Étude de cas : Habiter Paris, une ville mondiale } \\
\hline \multirow{5}{*}{$\begin{array}{l}\text { Séances } \\
\text { 1- Paris, une } \\
\text { métropole qui } \\
\text { concentre les fonctions }\end{array}$} & Activités & \begin{tabular}{|c|} 
Compétences \\
\end{tabular} \\
\hline & $\begin{array}{l}\text { Lecture par le professeur du sonnet } \\
\text { de J. Roubaud « Canal Saint-Martin » } \\
\text { (1999, p. .108). } \\
\text { Relecture silencicuse. }\end{array}$ & $\begin{array}{l}\text { Mise en ceuvre d'une démarche de compréhension : } \\
\text { identification et mémorisation des informations } \\
\text { importantes. } \\
\text { Mise en ceuvre efficace du décoodage. }\end{array}$ \\
\hline & $\begin{array}{l}\text { Travail individuel: les élèves doivent } \\
\text { dessiner le lieu du poème et reprendre } \\
\text { les différents élements du paysage, sous } \\
\text { forme dunue carte postale (voir travaux } \\
\text { de Gaujal, 2016). }\end{array}$ & $\begin{array}{l}\text { Comprehension du texte, se localiser dans l'espace, } \\
\text { analyse de paysage par le texte. }\end{array}$ \\
\hline & $\begin{array}{l}\text { Ces représentations initiales sont } \\
\text { alors confrontés à des représentations } \\
\text { spatiales grâce à une localisation des } \\
\text { lieux du pooène sur Google map et sur } \\
\text { Street view. }\end{array}$ & Apprentissage de l'utilisation de ces deux outils. \\
\hline & $\begin{array}{l}\text { Travail de groupe : localisation } \\
\text { d'autres lieux pour mettre en evidence } \\
\text { les fonctions économiques, sociales, } \\
\text { politiques et culturelles de Paris. } \\
\text { Construction d'une légende et de son } \\
\text { schéma. Voir figure } 2 \text {. }\end{array}$ & $\begin{array}{l}\text { Apprentissage du langage cartographique. } \\
\text { Découverte des caractéristiques d' une métropole } \\
\text { mondiale par un jeu de changement d'échelle. }\end{array}$ \\
\hline \multirow[t]{3}{*}{$\begin{array}{l}\text { 2- Habiter Paris, } \\
\text { une expérience } \\
\text { sensible }\end{array}$} & $\begin{array}{l}\text { Lecture du corpus de façon } \\
\text { individuelle. } \\
\text { Pratique de la lecture à voix haute. }\end{array}$ & $\begin{array}{l}\text { Autonomie dans le choix de la lecture d'un extrait } \\
\text { selon ses goûts. }\end{array}$ \\
\hline & $\begin{array}{l}\text { Lecture méthodique : repérer les } \\
\text { cléments du paysage, les ambiances, } \\
\text { travail sur r'́nonciation, sur les images, } \\
\text { travail sur la forme (ode, sonnet, } \\
\text { quinine) }\end{array}$ & $\begin{array}{l}\text { Prise en compte des groupes syntaxiques, des marques } \\
\text { de ponctuation et versification. } \\
\text { Sensibilisation aux effets esthétiques d'un texte. }\end{array}$ \\
\hline & $\begin{array}{l}\text { Travail de comparaison au brouillon } \\
\text { en groupe classe. }\end{array}$ & $\begin{array}{l}\text { Écrits de travail pour formuler des impressions de } \\
\text { lecture, émettre des hypothéses, articuler des idées, lister. }\end{array}$ \\
\hline \multirow[t]{4}{*}{$\begin{array}{l}\text { 3- Habiter Paris, } \\
\text { une expérience de la } \\
\text { mobilitíe }\end{array}$} & $\begin{array}{l}\text { Lecture de « Sunday, mein } \\
\text { Oberkampp ». } \\
\text { Travail de groupe : localiser sur } \\
\text { Google map et refaire le parcours sur } \\
\text { Street view, observer les changements } \\
\text { des magasins. }\end{array}$ & Lire une image, changement d'échelles. \\
\hline & $\begin{array}{l}\text { Travail d'écriture créative : Travail } \\
\text { de groupe. Choisir une autre rue, } \\
\text { parcours sur Street view. Construire la } \\
\text { carte du parcours choisi. }\end{array}$ & $\begin{array}{l}\text { Mise en couvre d'une démarche de production de } \\
\text { textes : convoquer un univers de référence, un matériau } \\
\text { linguistique. }\end{array}$ \\
\hline & Choix de la forme et justification. & $\begin{array}{l}\text { Pratique du brouillon. } \\
\text { Connaissance sur la langue (orthographe, ponctuation, } \\
\text { organisateurs du discours). }\end{array}$ \\
\hline & Finalisation du carnet. & $\begin{array}{l}\text { Entrainement à l'écriture sur ordinateur. } \\
\text { Mise en page des poèmes. } \\
\text { Lecture du texte à haute voix pour l'évaluer devant } \\
\text { pairs et enseignant. }\end{array}$ \\
\hline
\end{tabular}




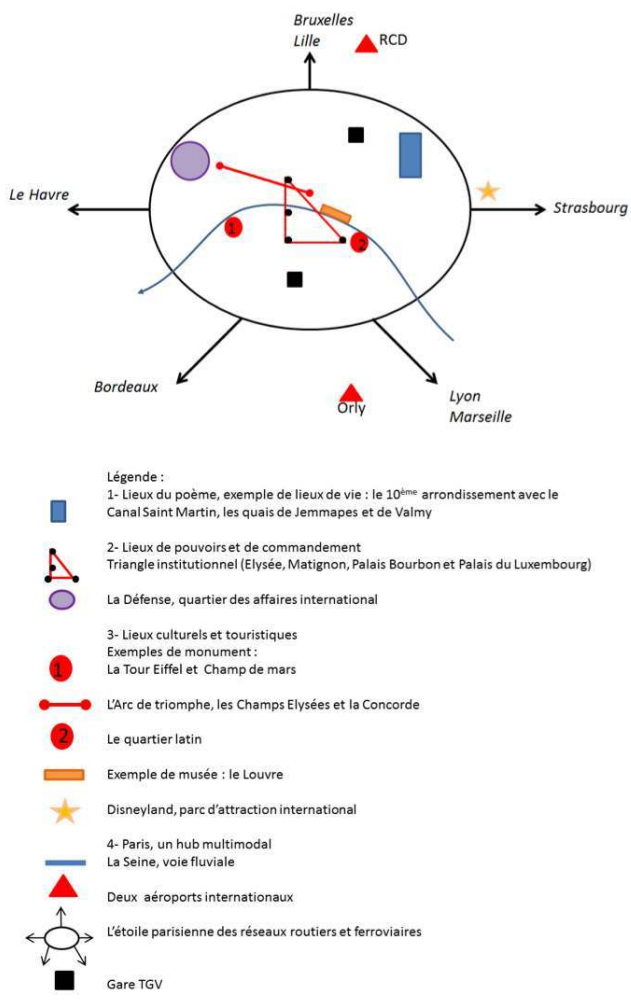

Cette séquence se situe au-delà d'une complémentarité disciplinaire; elle propose une interdisciplinarité, dans la mesure où le géographe a besoin du poème comme expérience sensible du territoire et le littéraire, de la géographie pour mieux appréhender la complexité du poème. Il s'agit alors de lire et de travailler des textes " contemporains", pour montrer que la poésie est un art vivant et dynamique. Ce va-et-vient entre les deux disciplines participe à une meilleure compréhension du monde et de soi-même car il permet aux élèves de construire des savoirs, de décloisonner leurs compétences pour réaliser un travail créatif. Ainsi, ils réfléchissent sur leur positionnement spatial et construisent leur être au monde, sur une singularité qui se nourrit du rapport à la langue, comme objet linguistique et culturel et aux différents langages (visuels, scientifiques, etc.). Dans ce protocole pédagogique, l'élève élabore son propre cheminement, que cela soit par des travaux de lecture, d'écriture, de dessin, pour passer du territoire à l'espace du poème. En effet, il s'agit pour l'élève de voir comment un espace peut être décrit objectivement par les outils de la géographie; puis par la lecture, d'appréhender comment des poètes réutilisent des données objectives pour rendre compte d'une perception subjectivée : le poème construit un nouveau paysage et la spécificité du poème est de dépasser l'association de signifiés. La signifiance, résultat d'une subjectivation maximale par l'utilisation du vers, montre autre chose qu'une carte, une photographie, plus près du ressenti. Il nous a alors paru intéressant de demander aux élèves de concevoir l'habiter dans cette double démarche : en saisir les éléments objectifs grâce à des outils géographiques, construire un espace mental abstrait et restituer un espace vécu subjectivé par un travail sur la langue. La production de l'élève doit rendre compte à la fois d'un apprentissage et d'un imaginaire géographiques, que le travail en lecture/ écriture poétique devrait vectoriser. 
38 Si ce travail est réalisé dans un cadre disciplinaire normé, après avoir acquis des savoirs géographiques et linguistiques, l'élève peut s'affranchir du cadre pour produire un poème, libérant la subjectivité.

39 Enfin la manipulation de globes virtuels et le travail en groupe permettent d'acquérir des compétences sociales et civiques. L'utilisation de ces «terres virtuelles» soulève de nombreux problèmes, comme la confusion entre le visible, le réel et le vrai (Genevois, 2008) ou encore les objectifs mercantiles de ces outils où « l'innovation vise à maintenir la position hégémonique de Google dans sa mission d'organiser l'information à l'échelle mondiale et de la rendre universellement accessible et utile » (Joliveau, 2013). Ce travail permet donc de faire réfléchir sur la nature de ces outils et de participer au développement de l'esprit critique. De plus, le travail en groupe favorise un certain nombre de savoirs être : respect de la parole, écoute, recherche du consensus.

\section{Conclusion}

40 Le géographe et le poéticien appréhendent de manière différente le poème, mais la double lecture permet de faire des ponts entre les deux communautés discursives. Le géographe grâce au langage poétique peut capturer les liens qui unissent l'homme à l'espace, point central de la notion d'habiter. Au contact de la géographie, le poéticien saisit la matérialité de la ville. Le géographe circonscrit, modélise, l'espace de manière objective. Le poète, par le travail sur le langage, produit une subjectivation radicale. Il éclaire la matérialité de la ville autrement. Confronter ces deux représentations de la ville, dans des communautés discursives différentes, permet d'avoir une approche plus complexe du paysage, que nous définissons comme "espace vécu, perçu et ressenti». Quant à l'approche interdisciplinaire, elle facilite ce va-et-vient entre matérialité et idéel. Habiter est à la fois un concept géographique et poétique parce qu'il relève de l'expérience. Les deux disciplines peuvent s'enrichir l'une l'autre parce que la géographie aide à voir, à comprendre l'espace et la pratique du langage poétique permet de modifier cette perception de l'espace pour se l'approprier. Le langage poétique permet au géographe d'accéder à la subjectivité du paysage, ce que les outils informatiques n'autorisent pas encore. Par cette approche interdisciplinaire, nous nous approchons donc d'une « poétique de la Terre » célébrée par A. Berque (2014) ou d'une géopoétique du consensus dépassant les débats épistémologiques qui animent les deux disciplines sur la place du phénoménologique.

41 En effet, M. Collot distingue deux définitions de la géopoétique, l'une énoncée par M. Deguy et l'autre par K. White ; le premier voit dans la poésie un art du langage, résultat de ce rapport à l'espace, le poème serait une mise en mots de l'espace alors que le second envisage le poème comme une expérience de l'espace mise en mots ; l'espace serait donc premier. Cette dualité se retrouve dans les débats des géographes dont A. Berque (2000) s'est fait l'écho. Ce dernier propose "une médiance ", où la dimension matérielle et la dimension idéelle de l'espace ne seraient plus opposées. Ce travail interdisciplinaire propose une "géopoétique ", à la croisée de toutes ces réflexions, en choisissant une démarche trajective, dans l'idée que la réalité n'est ni complètement objective, ni complètement subjective, qui forme un système complexe à la fois écologique, technique et symbolique indispensable à l'existence. Comme la géographie scolaire tend plutôt à l'objectivité, et la poésie à la subjectivité, l'alliance des deux approches permet de trouver cette médiance. 


\section{BIBLIOGRAPHIE}

BERNIÉ, J.-P. (2002). «L'approche des pratiques langagières scolaires à travers la notion de "communauté discursive" : un apport à la didactique comparée? ». Revue française de pédagogie 141, p. 77-88. En ligne : https://www.persee.fr/doc/rfp_0556-7807_2002_num_141_1_2917.

BERQUe, A. (2000) [1990]. Médiance. De milieux en paysages. Paris : Belin.

BERQUE, A. (2014). Poétique de la Terre. Paris : Belin.

Collot, M. (2005). Paysage et poésie. Du romantisme à nos jours. Paris : J. Corti.

COLlot, M. (2014). Pour une géographie littéraire. Paris : J. Corti.

DESBois, H., GERVAIS-LAMBONY, P. \& MUSSET, A. (2016). « Géographie : la fiction “au cœur" ». Annales de géographie 709-710 (3), p. 235-245. En ligne : https://www.cairn.info/revue-annales-degeographie-2016-3-page-235.htm\#.

DUPUY, L. \& PUYO, J.-Y. (dirs.) (2015). De l'imaginaire géographique aux géographies de l'imaginaire. Pau : Presses universitaires de Pau et des Pays de l'Adour.

GAUJAL, S. (2016). Une géographie à l'école par la pratique artistique. Thèse en géographie, Université Paris Diderot.

GENEVOIS, S. \& JOUNEAU-SION, C. (2008). « Utiliser les "globes virtuels" pour enseigner la géographie de la France ». L'Information géographique 72 (3), p. 81-93. En ligne : https://www.cairn.info/revue1-information-geographique-2008-3-page-81.htm.

JAUBERT, M. (2007). Langage et construction de connaissances à l'école. Pessac : Presses universitaires de Bordeaux.

JoliveAu, T. (2013). « Chacun sa carte ? Le nouveau Google Maps ». M@ppemonde 110. En ligne : https://mappemonde-archive.mgm.fr/num38/internet/int13201.html.

LAZZARotTI, O. (2006). Habiter. La condition géographique. Paris : Belin.

LHÉRÉTÉ, H. (2015). « Les pouvoirs de l'imaginaire ». Sciences humaines 273 (8), p. 21.

MANOLA, T., BAILLY, É. \& DURET, H. (2017). «Les ateliers-promenades : des expériences sensibles (paysagères) habitantes aux micro-interventions urbaines ». Projets de paysage 15. En ligne : https://www.projetsdepaysage.fr/ les_ateliers_promenades_des_exp_riences_sensibles_paysag_res_habitantes_aux_micro_interventions_urbaines

MESCHONNIC, H. (1982). Critique du rythme. Anthropologie historique du langage Lagrasse : Verdier.

PINSON, J.-C. (1995). Habiter en poète. Essai sur la poésie contemporaine. Seyssel : Champ Vallon.

Sтоск, M. (2004). « L'habiter comme pratiques des lieux géographiques ». EspaceTemps.net. En

ligne : https://www.espacestemps.net/articles/habiter-comme-pratique-des-lieuxgeographiques.

VERGNOLLE-MAINAR, C. (2011). La géographie dans l'enseignement. Une discipline en dialogue. Rennes : Presses universitaires de Rennes. 


\section{Recueils poétiques}

BARBARANT, O. (2015). Odes dérisoires et autres poèmes. Paris : Gallimard.

MARTEAU, R. (2012). Écritures. Seyssel : Champ Vallon.

RÉDA, J. (2004). L'adoption du système métrique, poèmes 1999-2003. Paris : Gallimard.

RITMAN, S. (2017). Ta résonance, ma retenue. Saint-Benoit-en-Sault : Tarabuste.

ROUBAUD, J. (1999). La Forme d'une ville change plus vite, hélas, que le cœur des humains. Paris :

Gallimard.

ROUBAUD, J. (2014). Octogone. Paris : Gallimard.

ROUZEAU, V. (2018). Sens averse. Paris : Éd. La Table ronde.

\section{NOTES}

1. "Sonnet X Canal Saint-Martin », "Sonnet XI Sunday, mein Oberkampf », La forme d'une ville change plus vite, hélas, que le cour des humains (Roubaud, 1999, p. 108-109) ; " Marronniers, place Fontenoy ", "Gamines, rue Sorbier ", L'adoption du système métrique (Réda, 2004, p. 100 et p. 103-104) ; "Une rose sauvage est encore au sommet », "Le coucou revenu mêle au chant de la grive ", Écritures (Marteau, 2012, p. 165 et p. 188) ; « Canal Saint-Martin (vers l'Hôtel du Nord, par exemple) », Octogone (Roubaud, 2014, p. 27-28), recueil dans lequel on trouve une réactualisation de «Sunday, mein Oberkampf » ( «Sunday, mine Oberkampf. Dix ans après », ibid., p. 29); "Chant de la porte Saint-Martin », Odes dérisoires et autres poèmes (Barbarant, 2015, p. 151-152); «l'eau de l'Oise monte sur les berges", Ta résonance, ma retenue (Ritman, 2017, p. 292) ; «Le chemin du peuplier seul va-t-il rester dans ta mémoire », Sens averse (Rouzeau, 2018, p. 97). Les poèmes de R. Marteau, S. Ritman et $\mathrm{V}$. Rouzeau sont sans titre, le premier vers est indiqué.

2. Nous ne référons pas ici à l'ouvrage de J.-C. Pinson, Habiter en poète, qui s'inscrit dans une démarche philosophique pour qui habiter équivaut à exister (1995, p. 26). J.-C. Pinson définit trois types de poètes: le poète-philosophe, le poète philologue et le poète-aède qui subsument la réalité du paysage. Nous essayons de penser un poète géographe, et le concept d'habiter est repris à 0 . Lazzarotti (2006) et à M. Stock (2004).

3. Un Système d'information géographique (SIG) est un logiciel informatique capable d'organiser et de présenter des données alphanumériques spatialement référencées. Il permet d'acquérir, d'organiser, de gérer, de traiter et de restituer des données géographiques sous forme de plans et cartes.

4. D’où l'expression de «nouveau lyrisme " pour caractériser la poésie contemporaine. Voir à ce propos M. Collot (2005, p. 15) : «l'énonciation lyrique, en première personne, correspond à la focalisation du paysage sur le point de vue d'un sujet ».

5. Ce balancement est indiqué dans le tableau par un changement de couleurs: en noir les activités et compétences appartenant à la communauté discursive poétique et en bleu celles de la géographie. 


\section{RÉSUMÉS}

Cet article réfléchit au concept d'habiter de manière interdisciplinaire. Il interroge un corpus de poèmes en vers contemporains qui intègrent le cadre spatial parisien. Qu'apportent les outils du géographe à la lecture des textes? Qu'apporte le poéticien à la compréhension de l'espace vécu? Nous avons voulu montrer que le travail du géographe était d'abord un travail sur les signifiés dans les poèmes choisis du fait des expériences du paysage urbain, implicites dans l'écriture poétique. Ensuite, nous avons cherché à mettre en évidence des procédés de signifiance des poèmes qui permettaient d'infléchir ou de nuancer une première perception de la ville. Enfin, nous avons proposé une trame de séquence didactique pour l'acquisition de la notion d'habiter en classe de $6^{\mathrm{e}}$, de manière à ce que les élèves combinent des compétences en géographie et en poésie pour appréhender dans ses dimensions subjective et objective leur paysage habituel.

This article reflects on the concept of dwelling in an interdisciplinary way. It questions a corpus of contemporary poems in verse that fit into the Parisian space. What do the geographer's tools bring to the reading of texts? What does the poetician bring to the understanding of lived space? We wanted to show that the work of the geographer was first of all a work on the signifieds in the chosen poems because of the poets's experience of the urban landscape. Then, we sought to highlight processes of significance of the poems that allowed to inflect or to qualify a first perception of the city. Finally, we proposed a didactic sequence for the acquisition of the notion of dwelling in sixth grade, so that students combine skills in geography and poetry to grasp in its subjective and objective dimensions their usual landscape.

poem, geography, dwelling, verse, landscape, interdisciplinarity

INDEX

Mots-clés : poème, géographie, habiter, vers, paysage, interdisciplinarité

\section{AUTEURS}

\section{SANDRINE BÉDOURET-LARRABURU}

Université de Pau et des pays de l'Adour, ALTER, EA 7504, F-64000, France

\section{DAVID BÉDOURET}

ESPÉ de Toulouse, Université Toulouse Jean Jaurès/CNRS, Géode, UMR 5602, F-31000, France 